\title{
Journée des délégués tarifaires de la FMH du 26 avril 2018
}

\begin{abstract}
Urs Stoffela, Patrick Müller ${ }^{b}$, Susanne Christen ${ }^{c}$, Sabine Zehnder ${ }^{d}$, Christian Oeschger ${ }^{\mathrm{e}}$, Thomas Kessler
${ }^{a}$ Membre du Comité central de la FMH, responsable du département Médecine et tarifs ambulatoires; ${ }^{b} \mathrm{FMH}$, chef de division, division Médecine et tarifs ambulatoires; ${ }^{c}$ Dr méd., $\mathrm{FMH}$, experte, division Médecine et tarifs ambulatoires; ${ }^{\mathrm{a}} \mathrm{FMH}$, experte, division Médecine et tarifs ambulatoires; ${ }^{e} \mathrm{FMH}$, chef de projet, division Médecine et tarifs ambulatoires; ${ }^{f} \mathrm{FMH}$, expert, division Médecine et tarifs ambulatoires
\end{abstract}

Placée sous le thème "Le nouveau tarif TARCO prend forme», la première Journée des délégués tarifaires de l'année, qui s'est tenue le jeudi 26 avril 2018 à Berne, a compté plus de 100 participants. Le nombre de participants se stabilise à ce niveau élevé.

Le Dr Urs Stoffel a souhaité la bienvenue aux participants et ouvert la Journée des délégués tarifaires en faisant le point sur la situation en politique de santé. Il a présenté le contexte politique actuel en évoquant la deuxième intervention du Conseil fédéral dans la structure tarifaire TARMED au $1^{\text {er }}$ janvier 2018 et l'arrêt du Tribunal fédéral concernant la première intervention tarifaire du Conseil fédéral. Alors même que le Département fédéral de justice et police (DFJP) a remis en question le caractère approprié des deux interventions tarifaires, le Tribunal fédéral a décidé que le Conseil fé- déral pouvait poursuivre des objectifs politiques en plus de s'en tenir au caractère approprié. Ensuite, le Dr Urs Stoffel a donné des informations sur le rapport d'experts du Conseil fédéral et les différentes mesures visant à freiner la hausse des coûts. Il a poursuivi son tour d'horizon en informant que la nouvelle nomenclature TARCO avait été approuvée lors de l'Assemblée des délégués du 11 avril 2018 et que son adoption par la Chambre médicale était prévue pour le 2 mai 2018. Pour terminer, il a présenté le calendrier du projet TARCO. L'objectif est de soumettre la structure tarifaire

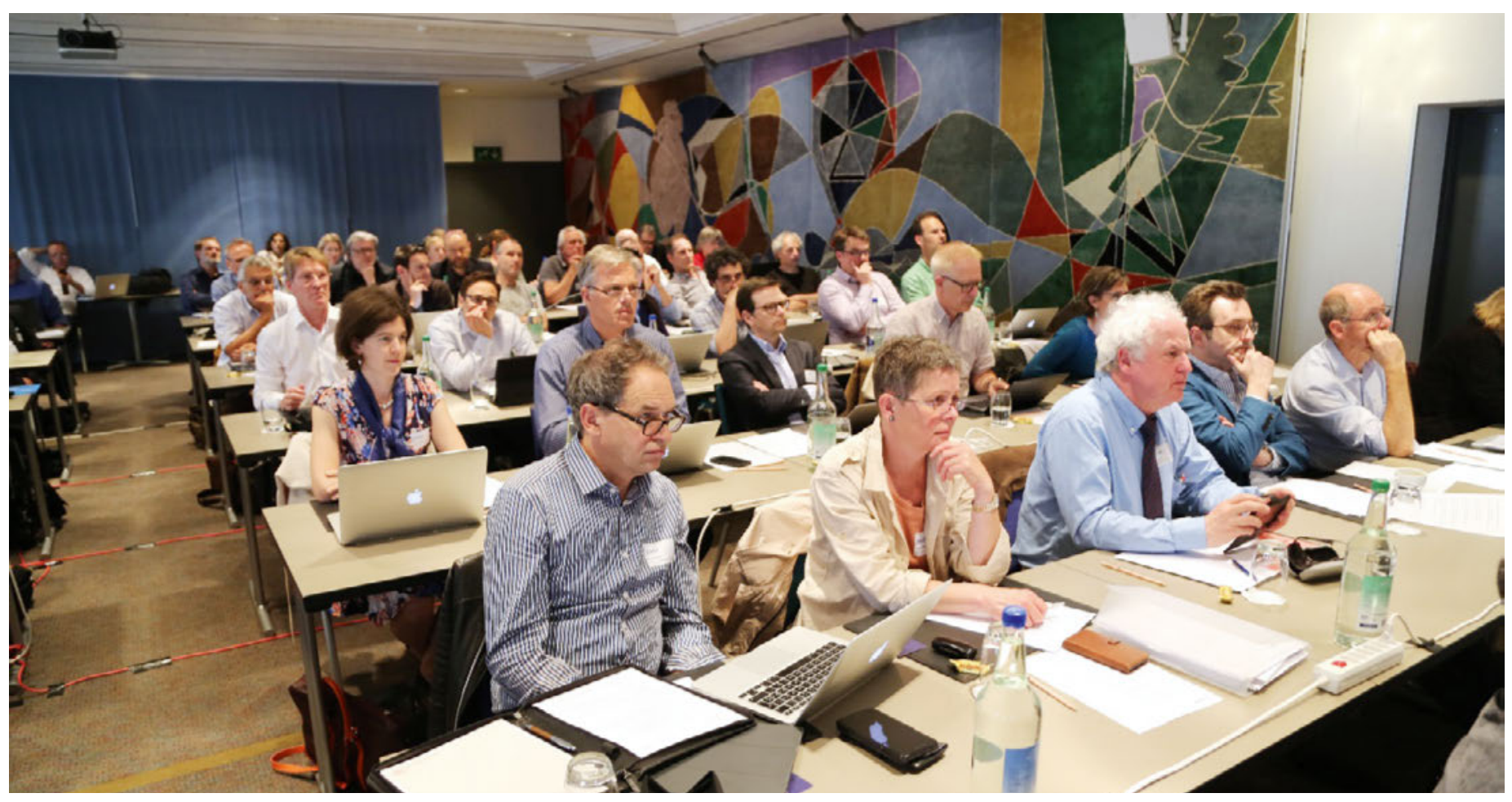

La Journée des délégués tarifaires de la FMH s'est tenue dans les locaux de l'Hôtel Bern à Berne. 


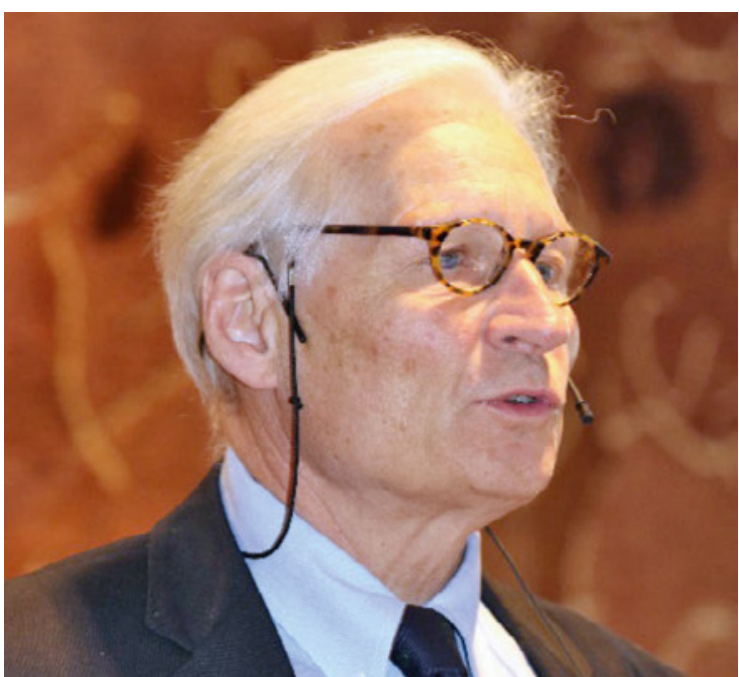

Le Dr Urs Stoffel souhaite la bienvenue aux participants.

révisée et négociée avec les partenaires tarifaires à la fin 2018 à l'approbation du Conseil fédéral.

\section{Nouveautés dans le domaine du laboratoire au cabinet}

La Dresse Susanne Christen a présenté les derniers développements dans le domaine du laboratoire au cabinet. La liste des analyses, un tarif officiel, fait à nouveau l'objet d'une révision. Le projet transAL de l'OFSP se poursuivra probablement jusqu'à fin 2020 et comprend l'élimination de positions d'analyses obsolètes et multiples, mais aussi de nouvelles tarifications. Le chapitre 5 de la liste des analyses, qui concerne le laboratoire au cabinet, sera également révisé. Deux motions sont actuellement discutées au Parlement. Elles

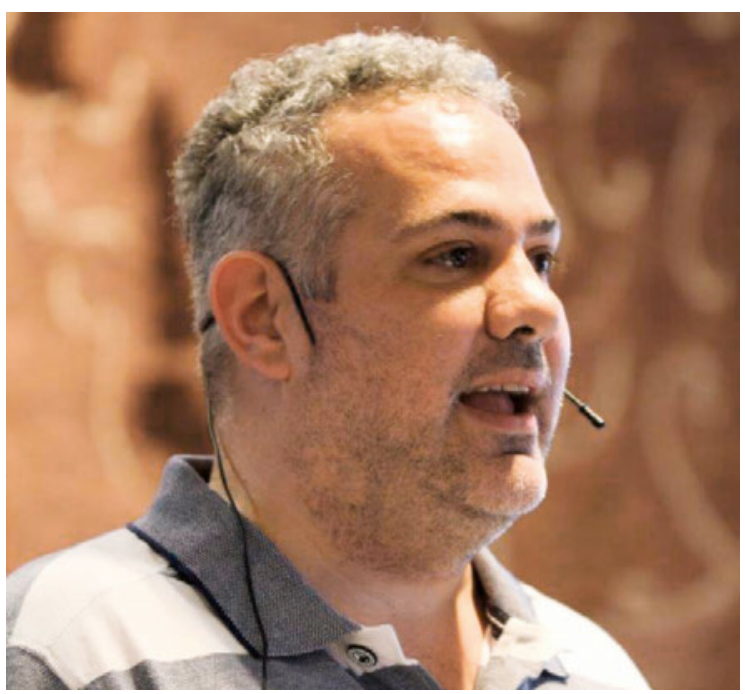

Thomas Kessler informe de l'état actuel des travaux du GT EAE.

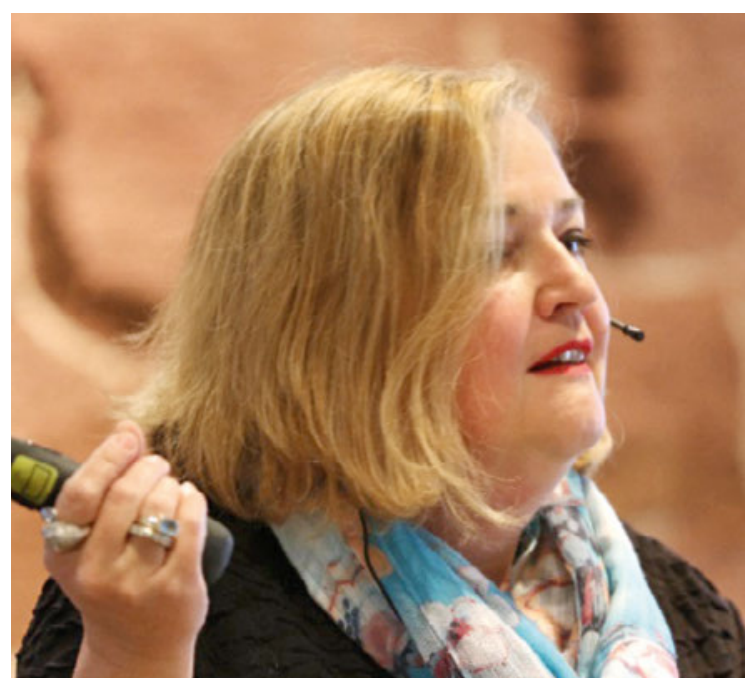

La Dresse Susanne Christen présente les derniers développements dans le domaine du laboratoire au cabinet.

prévoient que les tarifs pour les analyses par des laboratoires médicaux pourront à l'avenir être négociés par les partenaires tarifaires. La motion Kuprecht (17.3969) a été adoptée en novembre 2017 par le Conseil des Etats et la motion Hess (16.3193) en mars de cette année par le Conseil national. Les deux motions vont maintenant être traitées par le deuxième conseil. Enfin, la Dresse Christen a fait le point sur la situation actuelle à la Commission suisse pour l'assurance de qualité dans le laboratoire médical (QUALAB), sur la recertification de l'attestation de formation complémentaire du CMPR pour la pratique du laboratoire au cabinet médical (AFC-LP) et sur la Conférence de l'Union Suisse de Médecine de Laboratoire (USML) du 6 septembre 2018, qui aura pour sujet «La transformation digitale et les aspects scientifiques et politiques».

\section{GT EAE - état actuel des travaux}

Thomas Kessler a présenté l'état actuel des travaux du groupe de travail EAE (GT EAE). D’après l'art. 56 al. 6 LAMal, la situation initiale est la suivante: «Les fournisseurs de prestations et les assureurs conviennent d'une méthode visant à contrôler le caractère économique des prestations.» La FMH, santésuisse et curafutura se sont mis d'accord sur une méthode statistique pour identifier les médecins qui présentent des coûts très élevés sur la base d'un rapport final de Polynomics $\mathrm{SA}$, dans lequel la manière dont la méthode ANOVA peut être améliorée a été déterminée scientifiquement. Désormais, une analyse de régression à deux niveaux comprenant les variables de l'âge et du sexe, PCG, franchises (élevée/basse), séjour hospitalier l'année précédente, canton siège et groupe de spécialistes est mise en œuvre. L'objectif est d'augmenter sensible- 


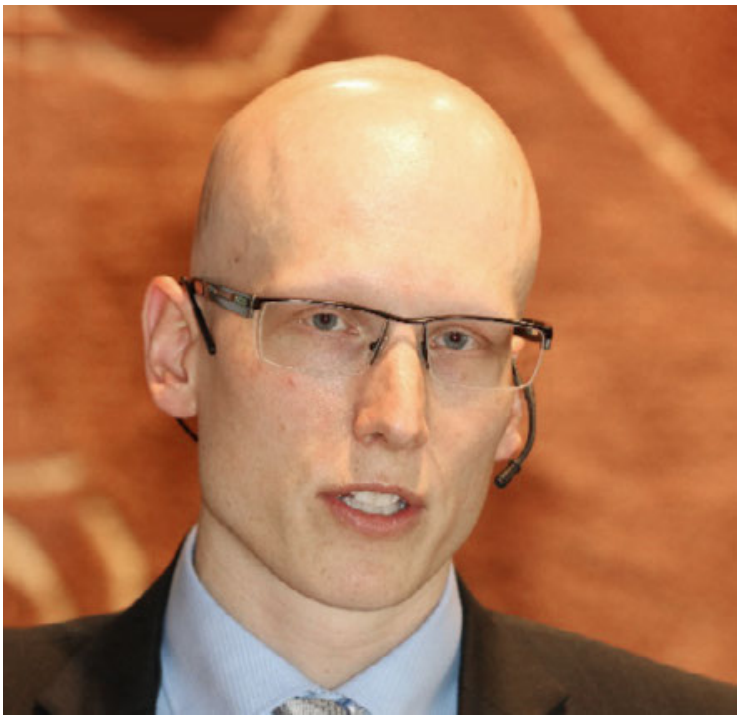

Patrick Müller donne des informations sur la deuxième intervention du Conseil fédéral dans le tarif TARMED.

ment la précision dans l'identification de médecins à la pratique inefficiente sur le plan économique. On remarquera à ce propos que la méthode d'identification statistique montre seulement qu'un médecin présente des coûts élevés par rapport au collectif comparatif. Une évaluation individuelle est ensuite indispensable pour déterminer si un médecin travaille de façon économique ou non. Une convention selon l'art. 56 al. 6 LAMal relative à la méthode d'identification statistique a été élaborée par la FMH, santésuisse et curafutura. Elle a été soumise au Comité central le 19 avril 2018. Le Comité central a décidé que la convention pourrait être soumise fin juin à l'Assemblée des délégués pour approbation définitive. Au terme de son exposé, T. Kessler a tiré les conclusions suivantes: «Avec la convention relative à la méthode d'identification statistique, nous faisons un pas dans la bonne direction, mais le potentiel d'amélioration dans le domaine du contrôle de l'économicité n'est de loin pas encore épuisé.»

\section{Deuxième intervention du Conseil fédé- ral dans le TARMED}

Patrick Müller a donné des informations sur la deuxième intervention du Conseil fédéral dans la structure tarifaire TARMED, qui a vu le jour suite à l'échec de la révision du TARMED négociée par les partenaires en 2016. Entre-temps, le tarif ordonné 1.09_BR est en vigueur depuis plus de 100 jours. Le moment est donc venu de tirer un bilan des premières expériences en la matière et des nombreuses questions des utilisateurs. Le besoin en renseignements et assistance concernant l'application du tarif TARMED ordonnée au $1^{\text {er }}$ janvier 2018 a été très élevé au cours des premiers mois de l'année. C'est pourquoi la ligne d'assistance a été renforcée en janvier 2018 à trois créneaux horaires hebdomadaires et en février et mars à deux créneaux horaires hebdomadaires. Pour le seul mois de janvier 2018, les collaborateurs de la division Médecine et tarifs ambulatoires ont mené environ 650 entretiens téléphoniques et répondu à 1100 courriels. Cela équivaut à environ 30 entretiens téléphoniques et 52 courriels par jour. Les principales questions concernent les thèmes des patients "nécessitant plus de soins", de l'application du facteur d'échelle pour le titre de formation postgraduée de médecin praticien et de l'application des prestations en l'absence du patient, désormais fortement différenciées et limitées.

Patrick Müller a par ailleurs parlé du groupe de travail mis en place par l'OFSP avec les partenaires tarifaires pour accompagner la deuxième intervention tarifaire. Il a notamment évoqué le monitorage exigé par l'ordonnance d'application. L'OFSP a envoyé aux partenaires tarifaires un catalogue d'exigences détaillé concernant le monitorage. Outre des chiffres précis sur la facturation (volumes et points tarifaires par titre de spécialiste et chapitre, taux de dépassement des limitations de volumes), il est également prévu d'établir des analyses qualitatives des effets de la nouvelle structure tarifaire. Pour terminer, P. Müller a encore évoqué la situation dans le domaine de l'assurance-accidents, invalidité et militaire. Comme aucun accord n'a été trouvé avec les assureurs-accidents CTM, la structure tarifaire 1.08_BR reste valable jusqu'à nouvel avis dans ce domaine, car la convention tarifaire existant entre la FMH et la CTM est encore en vigueur.

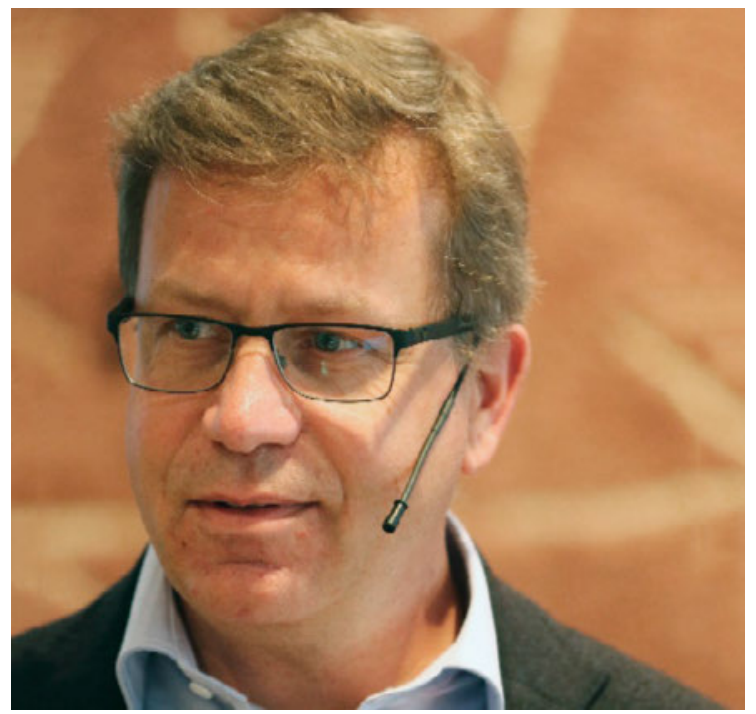

Le Dr Stefan Otto présente le sujet «L'ambulatoire avant I'hospitalier: la liste des interventions de la Confédération». 


\section{L'ambulatoire avant l'hospitalier: la liste des interventions de la Confédération}

Le Dr Stefan Otto a évoqué la liste des interventions de la Confédération "L'ambulatoire avant l'hospitalier». Il a ensuite procédé à une analyse du problème qui lui a permis de tirer les conclusions suivantes: il existe encore un potentiel de transfert considérable en Suisse. De plus, on constate la présence de fausses incitations tarifaires et d'un financement différent entre les secteurs ambulatoire et hospitalier. Actuellement, on se concentre sur les structures et les processus des fournisseurs de prestations orientées sur la prise en charge hospitalière; il n'existe pas de conditions uniformes pour tous les assurés en Suisse. Il est donc prévu d'élaborer une modification de l'ordonnance sur les prestations de l'assurance des soins (OPAS), qui entrera en vigueur le $1^{\mathrm{er}}$ janvier 2019. Elle vise deux objectifs. Premièrement, encourager la fourniture de prestations ambulatoires là où elle est indiquée d'un point de vue médical, mieux adaptée aux patients et moins coûteuse en ressources. Deuxièmement, assurer l'égalité de traitement pour tous les assurés AOS par une réglementation uniforme dans toute la Suisse. Le Dr Otto a indiqué que le potentiel de transfert pour certains types d'interventions est conséquent. Il a précisé que, selon une étude de l'OBSAN, les répercussions suivantes sur les coûts étaient attendues: potentiel de transfert d'environ 33000 cas, potentiel d'économie pour les cantons d'environ 90 millions $\mathrm{CHF}$ et pas de charge supplémentaire pour les assureurs-maladie.

\section{Projet TARCO - état actuel et dernières informations}

Après une courte pause, Sabine Zehnder et Christian Oeschger ont fait le point sur le projet TARCO. A la suite des dernières décisions concernant la nomenclature, les délégués du Cockpit ont approuvé la version définitive de la nomenclature TARCO le 15 mars 2018. Cette décision a été confirmée par l'Assemblée des délégués extraordinaire le 11 avril et la Chambre médicale le 2 mai 2018. Les deux experts de la FMH ont présenté les principales modifications aux délégués tarifaires.

Pour ne pas perdre de temps, la FMH a déjà repris au début de 2018 les négociations dans le domaine de la nomenclature avec les partenaires tarifaires curafutura, $\mathrm{H}+$ et CTM. A l'heure actuelle, les experts analysent conjointement les modifications effectuées dans TARCO et adressent leurs questions aux groupes de travail et sociétés de discipline concernées.

Les travaux, comprenant notamment l'intégration des propositions TARCO de la FMH dans la structure tarifaire partenariale, dureront au moins jusqu'à la fin août 2018. L'ats-tms SA ne sera donc pas en mesure de soumettre à la mi-juin 2018 un tarif commun au Conseil fédéral. Le dépôt conjoint de la structure tarifaire avec les partenaires tarifaires est maintenant prévu pour fin 2018.

Dans les semaines à venir, les experts de la FMH calculeront à nouveau les taux de coûts pour la prestation d'infrastructure et/ou de personnel (PIP) et les consigneront avec les positions tarifaires correspondantes.
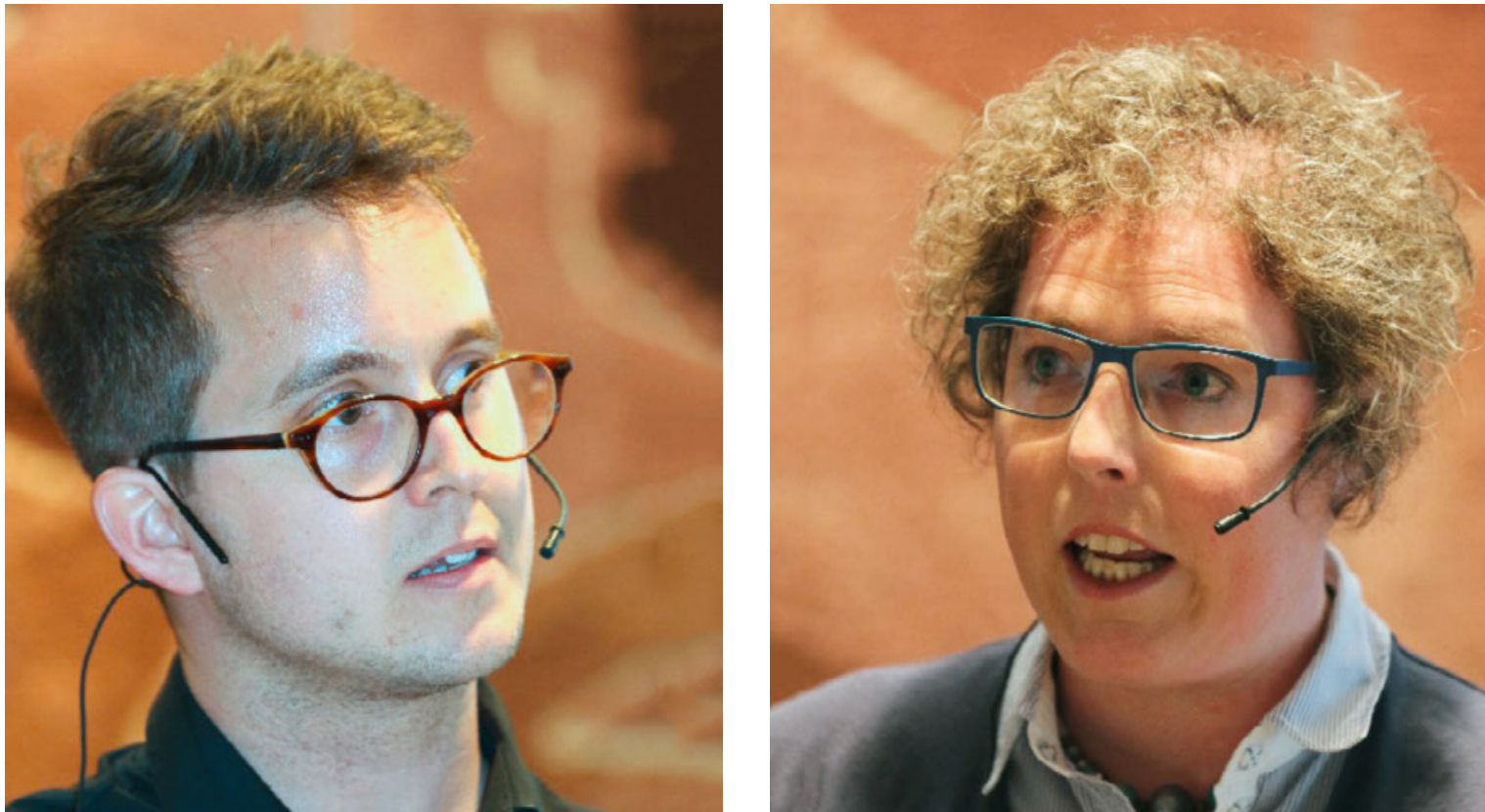

Christian Oeschger et Sabine Zehnder présentent les travaux actuels dans le cadre du projet TARCO. 


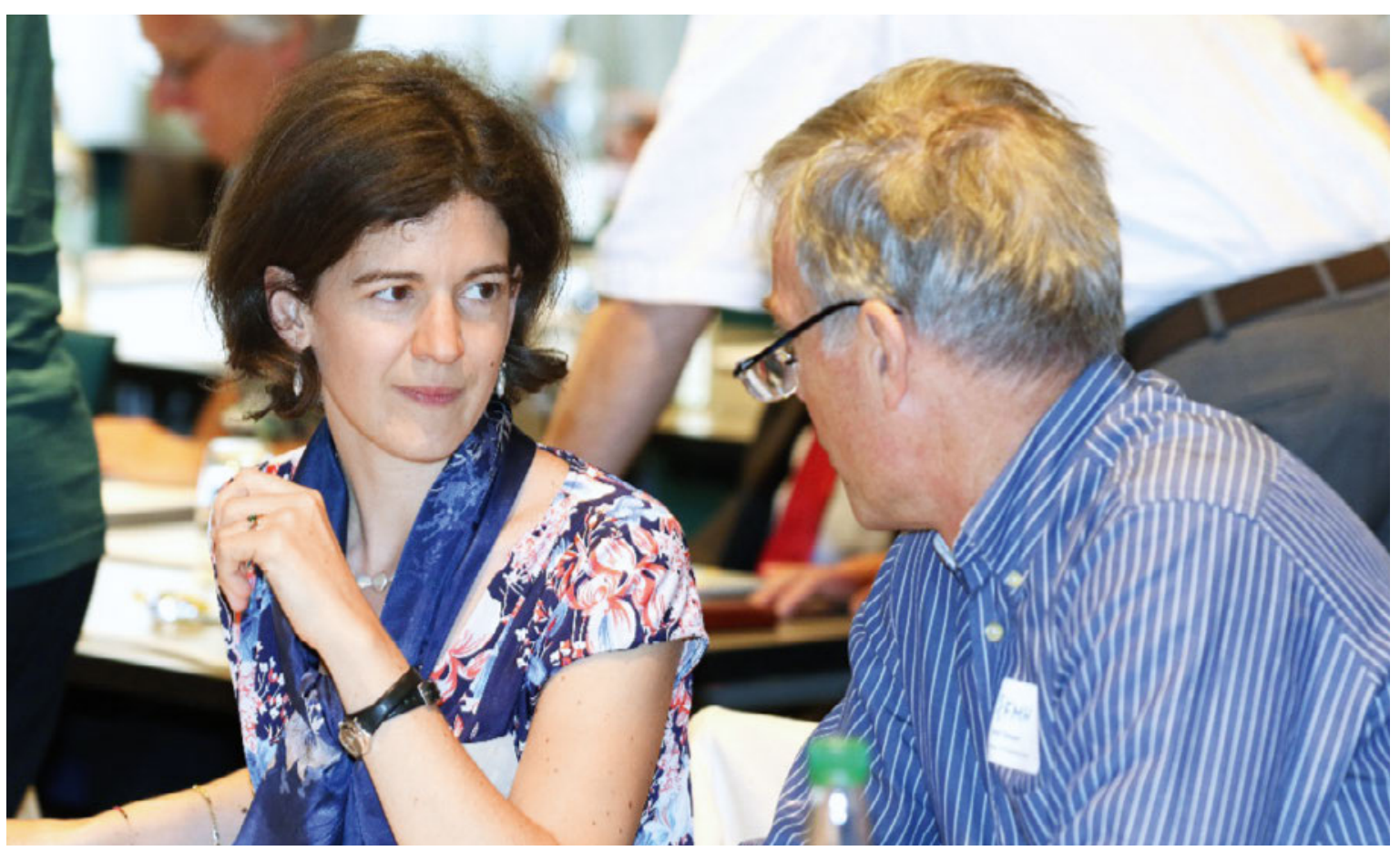

Les exposés ont suscité un vif intérêt de la part des participants à la Journée des délégués tarifaires.

Pour ce faire, le modèle de coûts KOREG est actuellement mis à jour en collaboration avec la Caisse des médecins. Dès que les taux de coûts INFRA définitifs auront été calculés, ils seront communiqués aux sociétés de discipline concernées.

En été, les sociétés de discipline seront encore une fois consultées par l'ats-tms SA au sujet des règles pour le cumul et les limitations. curafutura considère que des règles dans le tarif sont indispensables. La FMH accepte les règles si elles sont médicalement défendables; elle se montre ouverte à la discussion. En revanche, elle considère que les limitations du volume dans l'intention d'un rationnement des prestations sont inappropriées et vont à l'encontre des critères d'adéquation et d'efficacité applicables au traitement du patient. Fondamentalement, le principe de confiance s'applique pour le remboursement par l'AOS des prestations servant au diagnostic et au traitement d'une maladie. Du point de vue de la FMH, les assureurs disposent déjà, avec le contrôle individuel des factures, les procédures d'économicité et le tarifcontrolling, de suffisamment de moyens pour repérer les fournisseurs de prestations qui ne respectent pas le principe d'économicité.

\section{Rétrospective et perspective}

Pour conclure, le Dr Urs Stoffel a dressé un bilan et formulé le constat suivant: la pression politique sur le corps médical continue de s'accroître. Avec l'augmentation des primes, la population devient de plus en plus sensible. Il faut donc saisir l'opportunité qui se présente et prouver que le corps médical a la volonté de collaborer activement à une révision du tarif et de faire des compromis dans le cadre des négociations tarifaires. Combattons ensemble pour un tarif médical structuré de manière appropriée et fixé d'après les règles applicables en économie d'entreprise et, ainsi, pour un approvisionnement en soins de qualité dans l'intérêt des patientes et des patients.

\section{Crédits photo:}

Fotos: Andreas Weissenburger
FMH, division Médecine et tarifs ambulatoires

Baslerstrasse 47 CH-4600 Olten

Tél. 0313591230 Fax 0313591238 tarife.ambulant[at]fmh.ch
Vous trouverez tous les documents et la présentation PowerPoint de la Journée sur le site Internet de la FMH: www.fmh.ch $\rightarrow$ Tarifs ambulatoires $\rightarrow$ Journée des délégués tarifaires $\rightarrow$ 26 avril $2018 \rightarrow$ Présentation

La prochaine Journée des délégués tarifaires aura lieu le jeudi 27 septembre 2018 à l'Hôtel Ador à Berne. Notez d'ores et déjà cette date dans votre agenda! 